Check for updates

1 , London, UK

2 Association of Surgeons in Training, London, UK

3 Royal College of Surgeons of England, London, UK

Correspondence to: C Munro cmunro@bmj.com

Cite this as: BMJ2021;372:n659 http://dx.doi.org/10.1136/bmj.n659 Published: 12 March 2021

\title{
Covid-19 leaves surgical training in crisis
}

\author{
Urgent restoration of training is critical to the UK's future surgical workforce \\ Clara Munro, ${ }^{1}$ Josh Burke, ${ }^{2}$ William Allum, ${ }^{3}$ Neil Mortensen ${ }^{3}$
}

The pandemic has brought innumerable difficulties, some immediately obvious and others more insidious. Focus has correctly been on immediate risks to patients, healthcare workers, and the NHS. However, the extent of the detrimental effect of the pandemic on the provision of medical and surgical training has become increasingly clear. All trainees have had reduced experience in outpatient clinics, ward work, and multidisciplinary meetings because of redeployment to support the emergency response to covid-19.

Disruption has been particularly acute in procedure based specialties such as surgery. Operative training and other important competencies such as endoscopy have been severely affected. The cancellation of elective surgical services has decimated training opportunities over the past 12 months, with serious implications for recruitment and retention of the surgical workforce in the UK.

Review of trainee logbooks comparing 2019 and 2020 shows a 50\% reduction in operations with trainees as the primary operating surgeon. ${ }^{2}$ This creates a problem as patient outcomes and safety depend on surgeons achieving a high level of competence, including gaining experience under supervision. A lack of training opportunities slows the career trajectory of individual trainees and is also a major concern for the future adequacy of the surgical workforce. Appropriate numbers of surgeons must complete higher specialty training each year to ensure the NHS can provide a safe and timely service to patients.

Two groups of trainees have been particularly badly affected: early years trainees and those approaching the end of training. Redeployment of early years trainees, particularly in the first wave of the pandemic, and cancellation of elective surgery has denied them essential opportunities to develop the basic skills of surgical practice required to complete their core training. ${ }^{3}$ Among more senior trainees, $12 \%$ of those nearing the end of training were officially recognised as "delayed due to covid-19" in their latest annual review of competency progression, ${ }^{4}$ with this number expected to increase. As a result, the current cohort of senior trainees may require an extension to training and a bespoke training plan to achieve all competencies defined by the curriculum before they can progress to consultant level.

\section{Catch-up}

What can be done? Ideally, the provision of medical and surgical training should be at the centre of all healthcare delivery. The pandemic has left a substantial backlog of unmet surgical need, 5 and opportunities for training must not be lost during the catch-up in the coming months. We must all recognise the importance of training to the safe and effective management of conditions requiring surgery. Urgent restoration of training in operating theatres is essential for individual trainees, the future provision of services, and, most importantly, for patient care. Under current pandemic pressures, it can be easy to feel overwhelmed and frustrated, but supporting trainees to capitalise on all available opportunities is vital.

In November 2020, the Joint Committee on Surgical Training in collaboration with the Association of Surgeons in Training, British Orthopaedics Trainees' Association, and Confederation of Postgraduate Schools of Surgery published Maximising Training: Making the Most of Every Training Opportunity ${ }^{2}$ to help guide the recovery. It argues for national, regional, and local initiatives to ensure support for training programmes and trainers, and recognises the need for protected time for training within job plans. The report also argues for support for trainees who may have lost confidence during the pandemic or been affected by covid-19. Specific recommendations include providing elective training within the independent sector, individualising training trajectories, creating and expanding e-learning with webinars mapped to specialist curriculums, and developing simulation opportunities for learning both technical and non-technical skills.

These adjuncts to traditional hands-on training may be useful in both the short and medium term ${ }^{4}$ and provide an opportunity to review and refresh surgical training without compromising quality.

In the current climate of uncertainty, assigning an end date to the training emergency is difficult. We must also recognise that the workforce is already tired, demoralised, and running on reserves. A proactive approach to improving surgical training opportunities is an essential component of recovery, but the ability to provide these opportunities will depend on the patience and understanding of everyone within the NHS, including patients, surgical trainees, and their trainers. The situation is serious, and we must act to reverse the damage done to surgical training in the past 12 months. If there is no training today, there will be no surgeons tomorrow.

Competing interests: We have read and understood BMJ policy on declaration of interests and have no interests to declare.

Provenance and peer review: Commissioned; not externally peer reviewed.

COVID-STAR Collaborative.COVID-19 impact on surgical training and recovery planning (COVID-STAR): a cross-sectional observational study. Int J Surg 2021:105903. pmid: 33652133 
2 Joint Committee of Surgical Training, Association of Surgeons in Training, British Orthopaedics Trainees' Association, Confederation of Postgraduate Schools of Surgery. Maximising training: making the most of every training opportunity. 2021. https://www.jcst.org/key-documents/

3 Association of Surgeons in Training, British Orthopaedics Trainee's Association. CST redeployment and impact on progression. 2021. https://www.asit.org/news/asit-bota-statement-cst-redeployment-and-impact-on-progression/nwc11217

4 Health Education England. Guidance and principles for managing extensions to training during covid-19 (ARCP outcomes 10.1 and 10.2). 2020. https://healtheducationengland.sharepoint.com/Comms/Digita/SharedDocuments/Forms/Alltems.aspx?id=\%2FComms\%2FDigital\%2FShared Documents\%2Fhee.nhs.uk documents\%2FWebsite files\%2FCovid19\%2FCOVID19_Managing_Extensions_to_Training.pdf\&parent=\%2FComms\%2FDig

5 Carr A, Smith JA, Camaradou J, Prieto-Alhambra D. Growing backlog of planned surgery due to covid-19. BM/2021;372:n339. doi: 10.1136/bmj.n339 pmid: 33563590

This article is made freely available for use in accordance with BMJ's website terms and conditions for the duration of the covid-19 pandemic or until otherwise determined by BMJ. You may use, download and print the article for any lawful, non-commercial purpose (including text and data mining) provided that all copyright notices and trade marks are retained. 